\title{
A generalized PID controller for high-order dynamical systems
}

\author{
Günyaz Ablay ${ }^{1}$
}

\begin{abstract}
This paper introduces a generalized PID type controller for controlling high-order dynamical systems. An optimal generalized PID control design method is developed to provide a simplified high-order output feedback control design procedure and tunable response characteristics. The controller design procedure is reduced to the specification of the desired natural frequency and the solution of a polynomial equation. The control method is capable of providing a desired control performance under set-point and disturbance variations. The performance of the proposed control method is implemented on some unstable and nonlinear mechatronic systems to illustrate the robustness, effectiveness and feasibility of the method.

K e yw ords: control systems, PID control, mechatronic systems, optimization
\end{abstract}

\section{Introduction}

The PID controllers are an active research field and the most commonly adopted controller in industry because of their good performance and simplicity [1-3]. PID controller has a simple structure, tuning and implementation features, but it is only sufficient for the first and second order dynamical systems. For some higher-order systems, the application of PID controllers are limited to reduced-order models wherever possible [4]. But still, various advanced PID-based control schemes have also been designed to get good control performance for some higherorder dynamics while keeping the simplicity of the PID controllers. In this context, PID controller cascaded with filters [5,6], dead time compensation schemes [7], cascade control systems [8], ratio control systems [9], 2DOF PID controllers [10], the neural network algorithm [11] and fuzzy algorithm [12] based controllers, feedforward controllers [13], fractional-order PID [14, 15], event-based [16], nonlinear $[17,18]$ and predictive control [19] have been studied over the years. However, for controlling higher-order dynamical systems, the PID-type controllers are usually not applicable; if applicable, then the challenging issue in PID-based control approaches is to accomplish a proper combination of the controller block designs and the controller parameter tunings.

There are few generalized PID controllers for higherorder dynamical systems. An efficient generalized PID controller is known as coefficient diagram method (CDM) [20-22]. It is based on the characteristic ratio assignment [23], [24] and provides a good control performance under systems having parametric uncertainties [25-27]. The characteristic ratio assignment directly addresses the transient response control problem, eg, overshoot and rising time. However, it is not possible to shape the setpoint or disturbance rejection response with an adjustable setpoint-weight parameter as in the PID controllers. In addition, the establishment of the controller structure is not straightforward. The orders of the numerator and denominator of the controller must be designed with some trial-and-error approach, but this causes some practical design problems.

In this work, a 2-DOF generalized PID controller is proposed for controlling high-order dynamical systems. The proposed controller offers the advantages of PID controllers and the 2DOF control structures to control the $n$ th-order dynamical systems. That is, the $2 \mathrm{DOF}$ structure of the controller enables optimal set-point and disturbance rejection responses, and the PID-like structure of the controller provides design simplicity and tunable response characteristics. Compared with the other generalized PID-type controllers, the proposed controller has three distinguishable features: (i) it has a unique control structure including full features of the PID controller, (ii) it is designed systematically with only one parameter (ie, desired natural frequency $\omega$ ), and (iii) the controller has adjustable set-point weights for a desirable reference tracking and disturbance rejection performance. The proposed generalized PID controller can directly be designed for systems in any order with highly satisfactory and robust performances. The effectiveness and feasibility of the proposed controller is illustrated on some challenging linear and nonlinear mechatronic systems.

The paper is organized as follows. The proposed generalized PID controller is given in Section 2. Section 3 provides applications of the proposed controller to some challenging mechatronic systems, and Section 4 presents the conclusion of the study.

\section{A generalized PID controller}

In some control systems, a lower order controller may meet the given specifications, but in many control sys-

\footnotetext{
${ }^{1}$ Department of Electrical-Electronics Engineering, Abdullah Gül University, Kayseri, Turkey, gunyaz.ablay@agu.edu.tr
} 
tems, the existence of solution cannot be guaranteed. For these reason, a higher-order controller is usually required to provide a desired response. For instance, the PID controller is suitable for the first and second-order systems. For the third or higher order systems, state feedback based controllers are commonly designed with the cost of more measurement systems. An enhanced version of the PID controller, having performance features of the PID and state feedback controllers, can be designed for controlling the higher-order dynamical systems as presented below. Consider a dynamical system defined by

$$
G(s)=\frac{Y(s)}{U(s)}=\frac{a_{m} s^{m}+\cdots+a_{1} s+a_{0}}{b_{n} s^{n}+b_{n-1}+s^{n-1}+\cdots+b_{1} s+b_{0}},
$$

where the system is strictly proper $(m<n)$ and $b_{n}=$ 1. For the system (1), an enhanced PID-like controller similar to the state-feedback control approach can be defined as

$$
\begin{aligned}
u=k_{0} \varepsilon_{1} r-k_{0} y-k_{1} \dot{y} & \left.-\cdots-k_{(} n-1\right) y^{(n-1)}+ \\
& +k_{d}\left(\varepsilon_{2} \dot{r}-\dot{y}\right)+\int(r-y) \mathrm{d} t,
\end{aligned}
$$

where $r(t)$ is the reference signal, $\varepsilon_{1}$ and $\varepsilon_{2}$ are the set-point weights $k_{0}, \ldots, k_{n-1}, k_{d}$ and $k_{i}$ are the constant control gains. Suppose that the derivatives of $y(t)$ can be obtained through derivative filters, eg $y^{(n-1)}=$ $\left(s /(\tau s+1)^{n-1} y\right.$ where $\tau$ is the filter time constant and $s$ is the Laplace operator. Then, if we take the Laplace transform of the controller (2) and rearrange the terms, a generalized PID controller for $n$-th order system (1) is defined as

$$
\begin{aligned}
U(s) & =G_{r}(s) R(s)-G_{y}(s) Y(s), \\
G_{r}(s) & =\left(p_{2} b_{r} 2 s^{2}+p_{1} b_{r} 1 s+p_{0}\right) /\left(q_{2} s^{2}+q_{1} s\right), \\
G_{y}(s) & =\left(p_{n} s^{n}+\cdots+p_{1} s+p_{0}\right) /\left(q_{n} s^{n}+\cdots+q_{1} s\right),
\end{aligned}
$$

where $R$ is the set-point, $G_{r}$ is the fee dforward controller and $G_{y}$ is the stable feedback controller. The terms $q_{1}, \ldots, q_{n}, p_{0}, \ldots, p_{n}$, are the control coefficients to be designed; and $b_{r 1}, b_{r 2}$ are the set-point weights to be adjusted manually or numerically $\left(0<b_{r 1}, b_{r 2} \leq 1\right)$. If $n \leq 2$, the generalized PID controller (3) reduces to the classical PID controller. The block diagram of the proposed 2-DOF generalized PID controller (3) is seen in Fig. 1. With the 2-DOF structure, along with stabilization, the set-point tracking and disturbance rejection performances of the controller can be designed independently.

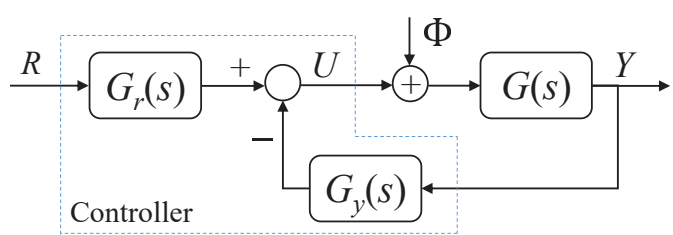

Fig. 1. Schematic diagram of the proposed controller
Then substituting the controller (3) into the system (1), the closed-loop system is written as

$$
Y(s)=\frac{G_{r}(s) G(s) \cdot R(s)+\Phi(s)}{\left(1+G_{y}(s) G(s)\right)},
$$

where $\Phi$ is the disturbance. The controller $G_{y}(s)$ with proper coefficients must first stabilize the system to have a desired closed-loop transfer function. Thus, if we define $G=A / B$ and $G_{y}=A_{c} / B_{c}$, then the characteristic equation of the closed-loop, $1+G_{y} G=0$, can be written as $B B_{c}+A A_{c}=0$. If a desired polynomial is given as $C(s)$ for given a set of desired poles, then the controller coefficients can be solved with pole placement approach by constructing the following polynomial equation

$$
B(s) B_{c}(s)+A(s) A_{c}(s)=C(s)
$$

This polynomial is known as Diophantine equation [28]. Matching the coefficients with respect to power of $s$ in (5) yields the following matrix equation

$$
\left[\begin{array}{cccccccc}
0 & 0 & \ldots & 0 & a_{0} & 0 & \ldots & 0 \\
b_{0} & 0 & \ldots & 0 & a_{1} & a_{0} & \ldots & 0 \\
b_{1} & b_{0} & \ldots & 0 & \vdots & \vdots & \ddots & \vdots \\
\vdots & \vdots & \ddots & \vdots & a_{m} & a_{m-1} & \ldots & a_{0} \\
b_{n-1} & b_{n-2} & \ldots & b_{0} & 0 & a_{m} & \ldots & a_{1} \\
b_{n} & b_{n-1} & \square & b_{1} & 0 & 0 & \square & a_{2} \\
\ddots & \ddots & \ddots & \vdots & \vdots & \vdots & \ddots & \vdots \\
0 & \ldots & b_{n} & b_{n-1} & 0 & 0 & \ldots & 0 \\
0 & \ldots & 0 & b_{n} & 0 & 0 & \ldots & 0
\end{array}\right]\left[\begin{array}{c}
q_{1} \\
q_{2} \\
\vdots \\
q_{n} \\
p_{0} \\
p_{1} \\
\vdots \\
p_{n}
\end{array}\right]=\left[\begin{array}{c}
c_{0} \\
c_{1} \\
\vdots \\
c_{n} \\
\vdots \\
c_{2 n}
\end{array}\right]
$$

where the matrix has a dimension of $(2 n+1)$ by $(2 n+1)$. The equation (6) can be written as $F K=C$, where the system matrix, controller vector and desired polynomial vector are defined as $F, K$ and $C$, respectively. Hence, a solution is obtained through $K=F^{-1} C$. For the equation (6), a solution always exists if $G=A / B$ is proper. A suitable solution for the Diophantine equation may not be found when $F$ is not a square matrix, which is the main problem of the reduced-order controllers such as classical PID and lower-order CDM. On the other hand, the proposed controller (3) has the order of the system so that a square matrix is always constructed to obtain suitable solutions for nth-order systems.

The closed-loop system stability can be achieved with suitable control parameter values. The pole placement approach can directly be used to calculate control coefficients. Namely, let us define a desired characteristic polynomial by

$$
C(s)=c_{2 n} s^{2 n}+\cdots+c_{1} s+c_{0} .
$$


The desired characteristic polynomial (7) can be constructed from various standard filter design polynomials [29], including Binomial, ITAE, and Butterworth-type polynomials and characteristic ratio assignments [24], $[30,31]$. If we consider the binomial polynomials, the coefficients in terms of natural frequency $\omega$ can be given by the formula

$$
(s+\omega)^{2 n}=\sum_{k=0}^{2 n} C(2 n, k) \omega^{2 n-k} s^{k},
$$

where $C(2 n, k)=(2 n !) /(k !(2 n-k) !)$ for $k=0,1, \ldots, 2 n$, and the desired coefficients of $(7)$ are $c_{k}=C(2 n, k) \omega^{2 n-k}$. The binomial transfer function poles provide fast response with some overshoot, so the set-point weights, $0<b_{r 1}, b_{r 2} \leq 1$, can be adjusted to eliminate the undesired overshoot.

The denominator of controller $G_{y}$ must have stable dynamics, which is desirable in terms of the integrity of feedback control system. For stable systems, a stable controller can always be found. However, for non-minimum phase unstable systems, the parity interlacing property (PIP) must be satisfied to obtain a stable controller. The PIP condition states that a necessary and sufficient condition for the existence of a stable stabilizing controller is that between each zero of the system on the RHP real axis (including infinity as a zero), there be an even number of poles [32]. For a stable controller, the natural frequency $\omega$ in (8) must be selected appropriately such that all the roots of controller denominator, found from $q_{n} s^{n}+\cdots+q_{1} s=0$, have negative real parts (one root is zero due to the integrator).

\subsection{Performance analysis}

The stability of closed-loop system can be evaluated through the roots of $C(s)$. The polynomial $C(s)$ is a Hurwitz polynomial if and only if all of its roots have negative real parts. The Lipatov and Sokolov conditions [33] are also very useful for quick checking the stability, which is given by

$$
\left.\left.\left.c_{(} i-1\right) c_{(} i+2\right) \leq 0.4655 c_{i} c_{(} i+1\right), i=1, \ldots, 2 n-2 .
$$

These stability conditions (9) also provides an effective way of producing robust polynomials. By considering the robust Hurwitz polynomials [34], the robustness of the controller under small parameter changes can be assessed. First, let us define the following parameter interval for all coefficients of the desired characteristic polynomial

$$
c_{i} \in\left[\alpha_{i}, \delta_{i}\right], \delta_{i} \geq \alpha_{i}>0
$$

then, the interval polynomial $\mathrm{C}(\mathrm{s})$ given by (7) and (10) is Hurwitz for all $c_{i} \in\left[\alpha_{i}, \delta_{i}\right]$, if

$$
\left.\left.\left.\delta_{(} i-1\right) \delta_{(} i+2\right) \leq 0.4655 \alpha_{i} \alpha_{(} i+1\right), \quad i=1, \ldots, 2 n-2 .
$$

Hence the proposed controller has robustness against small parameter variations according to above stability condition. The controller has the same robustness features of the characteristic ratio assignment and the CDM.

The disturbance rejection performance of the controller can easily be evaluated for step reference and step disturbance inputs, ie, $R(s)=r / s, \Phi(s)=981 / s$, such that the steady-state response is obtained as

$$
\lim _{s \rightarrow 0} s \frac{G_{r}(s) G(s) r / s+\phi / s}{1+G_{y}(s) G(s)}=r .
$$

Since both $G_{r}$ and $G_{y}$ include an integrator as seen in controller (3), the steady-state response of the closed-loop system will have zero error under the step disturbance input.

\subsection{Tuning the control parameters}

The control parameters can easily be tuned with natural frequency of the binomial polynomial (8). The setpoint weights, $b_{r 1}$ and $b_{r 2}$, can be adjusted to eliminate the undesired overshoot under step set-point and disturbance variations. These parameters can be adjusted manually or numerically. A numerical search algorithm can be performed to get optimal results. For this goal, the Nelder-Mead simplex algorithm [35] can be implemented for finding the optimal set-point weight values. The MATLAB's "fminsearch" function uses this algorithm to solve nonlinear optimization problems. To obtain suitable results, the ITAE performance index, IT AE = $\int_{0}^{\infty} t|e(t)| \mathrm{d} t$, can be used to get conservative controller settings. For example, a MATLAB/Simulink based algorithm provided in [36] can directly be utilized to find optimal set-point weight settings.

\section{Applications}

The various designs and control performance of the proposed controller are illustrated on some high-order and challenging practical systems including a three-cart system, the single-link flexible joint robot and the planar quadrotor system.

\subsection{Three-cart system}

The multi-DOF spring-mass-damper system is commonly used to model various systems, eg, robotic motion control. These systems can be unstable and vibrate at a certain natural frequency with a certain natural displacement, and thus they are difficult to control and operate [37]. The model of three-cart system as illustrated in Fig. 2 is given by

$$
\begin{aligned}
\dot{x}_{1} & =x_{2}, \\
\dot{x}_{2} & =\left(-f_{1} x_{2}-k x_{1}+k x_{3}+\alpha V\right) / m_{1}, \\
\dot{x}_{3} & =x_{4}, \\
\dot{x}_{4} & =\left(-f_{2} x_{4}-2 k x_{3}+k x_{1}+k x_{5}\right) / m_{2}, \\
\dot{x}_{5} & =x_{6}, \\
\dot{x}_{6} & =\left(-f_{3} x_{6}-k x_{5}+k x_{3}\right) / m_{3}, \\
y & =x_{5},
\end{aligned}
$$




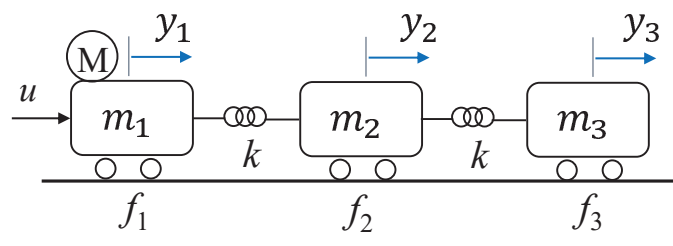

Fig. 2. The schematic diagram of three-cart system
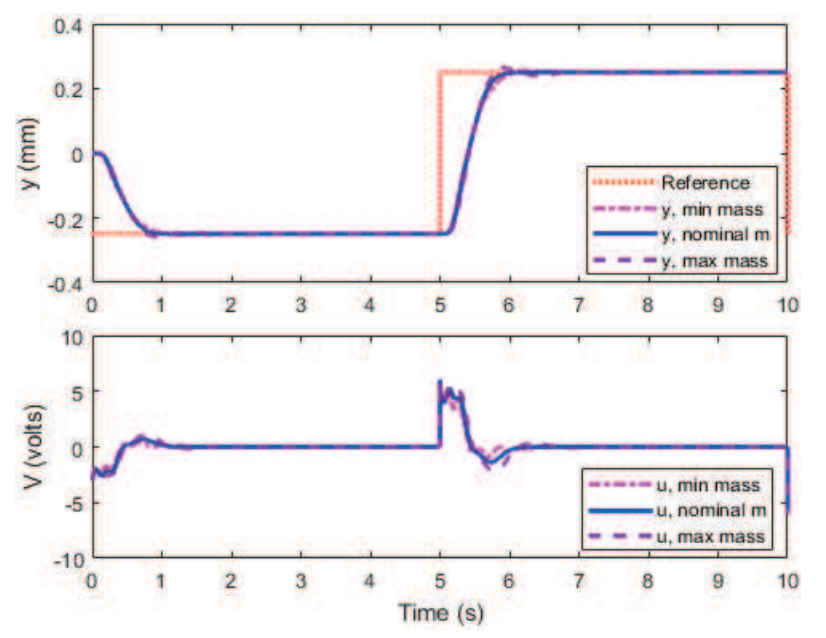

Fig. 3. The output and control signal responses of the three-cart system under cart mass variations

where $y(m)$ is the position of the third cart, $V(\mathrm{~V})$ is the motor control voltage, $m_{1}=1.6(\mathrm{~kg}), 0.75 \leq m_{2}, m_{3} \leq$ $1.25(\mathrm{~kg}), k=400(\mathrm{~N} / \mathrm{m}), f_{1}=0.09(\mathrm{Ns} / \mathrm{m}), f_{2}=f_{3}=$ $3.68(\mathrm{Ns} / \mathrm{m})$ and $\alpha=3.156$. The control goal is to design a controller so that the third cart can track a given step inputs as fast and accurately as possible without having any oscillations. The controller must be robust enough to deal with mass variations in the second and third carts. The motor voltage is limited by $|V| \leq 12$ volts. The system is unstable with a RHP pole. The classical PID controller cannot solve the mechanical oscillations of the system, so a higher-order controller is needed for providing a desirable performance.

Since the system order is $n=6$, the controller is written from (3)

$$
\begin{aligned}
G_{r} & =\frac{p_{2} b_{r 2} s^{2}+p_{1} b_{r 1} s+p_{0}}{q_{2} s^{2}+q_{1} s} \\
G_{y} & =\frac{p_{6} s^{6}+\cdots+p_{1} s+p_{0}}{q_{6} s^{6}+\cdots+q_{2} s^{2}+q_{1} s}
\end{aligned}
$$

Using the twelfth-order binomial polynomial for $\omega=25$ to have a stable feedback controller $G_{y}$, the control gains are found as $p_{0}=1.88 \times 10^{11}, p_{1}=9.06 \times 10^{10}, p_{2}=$ $1.39 \times 10^{10}, p_{3}=-5.43 \times 10^{8}, p_{4}=4.84 \times 10^{7}, p_{5}=$ $-2.02 \times 10^{6}, p_{6}=1.89 \times 10^{4}, q_{1}=2.54 \times 10^{9}, q_{2}=$ $1.15 \times 10^{8}, q_{3}=2.72 \times 10^{6}, q_{4}=3.76 \times 10^{4}, q_{5}=292.58$, $q_{6}=1$, and the set-point weights are selected as $b_{r 1}=$ 0.1 , and $b_{r 2}=0.1$, when the cart masses are $m_{2}=m_{3}=$ $1(\mathrm{~kg})$. Figure 3 shows the control performance under mass variations. The controller is designed for nominal masses $m_{2}=m_{3}=1(\mathrm{~kg})$, but these masses are variable due to the loads. It is seen from Fig. 3 that the controller provides a robust and highly satisfactory non-overshoot performance under $\pm 25 \%$ mass variations. The control voltage has very smooth variations without reaching the supply limits.

\subsection{A single-link flexible-joint robot}

Robot control is a quite challenging problem especially if the robot includes a flexible joint or link. Such flexibility produces nonlinearities and increases the system order, which results in vibration and low control performance. The equations of motion of the flexible-joint robot arm driven by a dc motor as shown in Fig. 4 is given by

$$
\begin{aligned}
& I \ddot{q}_{1}=M g l \sin q_{1}+k\left(q_{1}-q_{2}\right), \\
& J \ddot{q}_{2}=-k\left(q_{1}-q_{2}\right)=u, \\
& y=q_{1},
\end{aligned}
$$

where the experimental parameter values are taken from [12] as $M g l=0.8(\mathrm{Nm}), \quad I=0.03\left(\mathrm{kgm}^{2}\right), \quad J=$ $0.004\left(\mathrm{kgm}^{2}\right)$ and $k=31(\mathrm{Nm} / \mathrm{rad})$. The angular position of arm, $q_{1}$, is the measurable output. With linearization at origin, $i e, \sin \left(q_{1}\right) \approx q_{1}$, a transfer function is obtained as

$$
G(s)=\frac{Y(s)}{U(s)}=\frac{2.583 \times 10^{5}}{s^{4}+8810 s^{2}+2.053 \times 10^{5}} .
$$

The system has four imaginary poles, which is not possible to control with classical PID controllers. Since $n=4$, the controller is written from (3) as

$$
\begin{aligned}
G_{r}(s) & =\frac{p_{2} b_{r 2} s^{2}+p_{1} b_{r 1} s+p_{0}}{q_{2} s^{2}+q_{1} s} \\
G_{y}(s) & =\frac{p_{n} s^{n}+\cdots+p_{1} s+p_{0}}{q_{n} s^{n}+\cdots+q_{1} s}
\end{aligned}
$$

where for a stable $G_{y}$, the control gains are calculated from binomial polynomial (8) for $\omega=60$ as $p_{0}=16.5 \times$ $10^{8}, p_{1}=8.04 \times 10^{7}, p_{2}=4.98 \times 10^{6}, p_{3}=-9.936 \times$ $10^{4}, p_{4}=390.86, q_{1}=7.89 \times 10^{6}, q_{2}=92043, q_{3}=$ $480, q_{4}=1$, and the set-point weights are found from numerical optimization as $b_{r 1}=0.125$, and $b_{r 2}=0.12$. Figure 5 illustrates the reference tracking response of the controller. It is clear that the controller provides a fast, non-overshoot response and highly effective disturbance rejection performance.

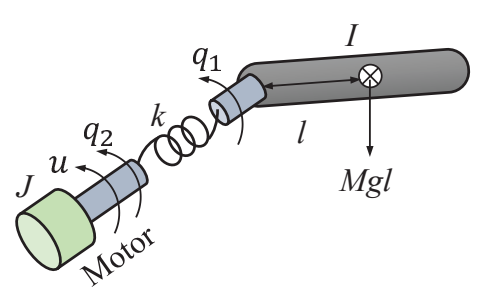

Fig. 4. A single-link flexible joint robot 

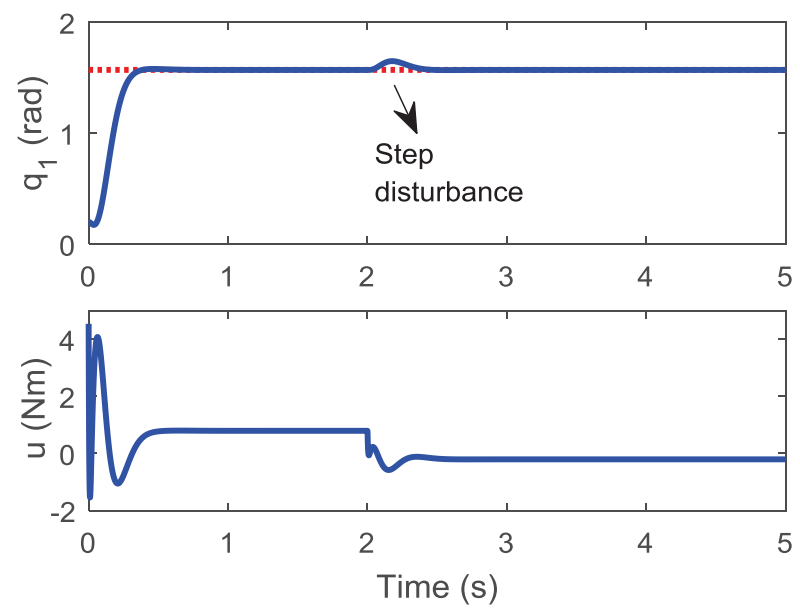

Fig. 5. Time response of the controlled system, (a) system output, (b) control signal

\subsection{Planar quadrotor}

Quadrotors have become very popular in recent years for various mobile applications [38]. They have nonlinear under-actuated dynamics, which causes some challenges in suitable controller designs. The equations of motion of a planar quadrotor flying in $y-z$ plane as illustrated in Fig. 6 is given by

$$
\begin{aligned}
& m \ddot{z}=-m g+u_{1} \cos \varphi, \\
& m \ddot{y}=-u_{1} \sin \varphi, \\
& l_{x x} \ddot{\varphi}=u_{2},
\end{aligned}
$$

where $m$ is the mass $(m=0.2 \mathrm{~kg}), I_{x x}$ is the inertia $\left(I_{x x}=0.1 \mathrm{kgm}^{2}\right), g=9.81 \mathrm{~m} / \mathrm{s}^{2}, u_{1}$ is the thrust force, $u_{2}$ is the moment about the $x$-axis, and the planar quadrotor pose is represented by $(y, z, \varphi)$. Linearization of the system around hover point, $z=z_{e}, y=y_{e}, \varphi=0$, $u_{1}=m g, u_{2}=0$, the transfer function of system can be obtained as

$$
\begin{aligned}
& Z(s) / U_{1}(s)=5 / s^{2}, \\
& Y(s) / U_{2}(s)=-98.1 / s^{4}, \\
& \varphi(s) / U_{2}(s)=10 / s^{2} .
\end{aligned}
$$

The controller can be designed independently for $z$ and $y$ positions while the system dynamics are highly nonlinear and coupled. Since we have $Y / U_{2}=Y / \varphi \cdot \varphi / U_{2}$ and $Y(s) / \varphi(s)=-9.81 / s^{2}$, a cascade controller can be designed to control the position $y$. Then, for time-varying smooth references, the controllers from (3) are designed to be

$$
\begin{aligned}
& U_{1}=\frac{p_{21} s^{2}+p_{11} s+p_{01}}{q_{21} s^{2}+q_{11} s}\left(Z_{r}-Z\right), \\
& \varphi_{r}=\frac{p_{22} s^{2}+p_{12} s+p_{02}}{q_{23} s^{2}+q_{13} s}\left(Y_{r}-Y\right), \\
& U_{2}=\frac{p_{23} s^{2}+p_{13} s+p_{03}}{q_{23} s^{2}+q_{13} s}\left(\varphi_{\varphi}\right),
\end{aligned}
$$

where $Z_{r}, Y_{r}$ are the references, $U_{1}$ is the position controller, $\varphi_{r}$ is the outer loop position controller, and $U_{2}$ is the inner loop attitude controller. Since the references are time-varying and smooth, all set-point weights are taken as one. The fourth-order binomial polynomial is used to calculate control gains $u_{1}, \varphi_{r}$ and $u_{2}$ when $\omega_{1}=8, \omega_{2}=4, \omega_{3}=40$, respectively. The control gains are calculated as $p_{01}=819, p_{11}=409.6, p_{21}=76.8$, $q_{11}=32, q_{21}=1$ for $u_{1}, p_{02}=-26, p_{12}=-26$, $p_{22}=-9.78, q_{12}=16, q_{22}=1$, for outer loop position controller $\varphi_{r}$, and $p_{03}=256000, p_{13}=25600$, $p_{23}=960, q_{13}=160, q_{23}=1$ for inner loop attitude controller $u_{2}$. Figure 7 illustrates the tracking performance and control inputs of the designed controller for $Z_{r}=4 \sin (t)$ and $Y_{r}=0.4 \sin (0.5 t)$. Even though the system is highly nonlinear, it is clear that fast and very good tracking results are obtained.

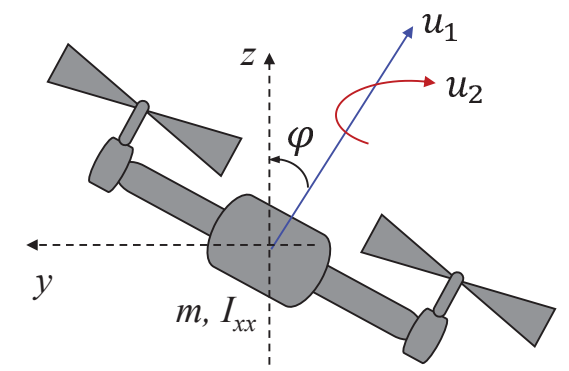

Fig. 6. Schematic diagram of a planar quadrotor flying about the hover state

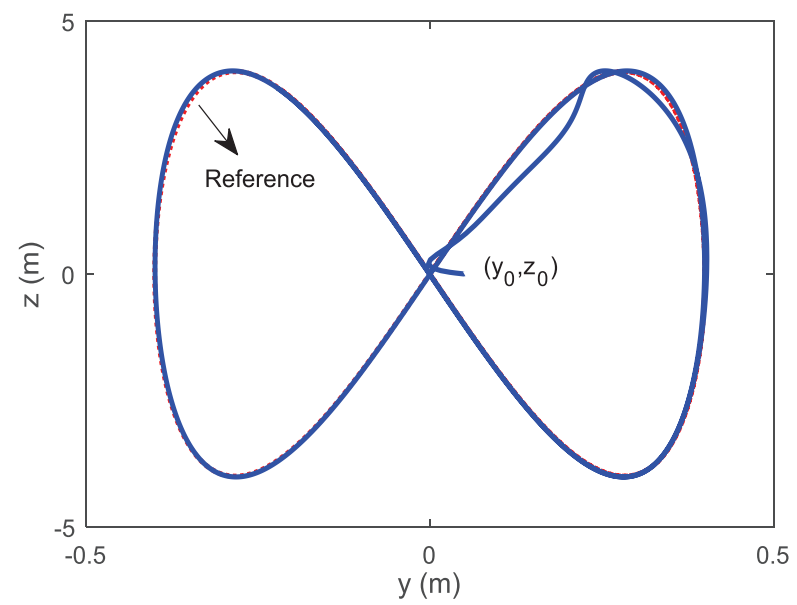

Fig. 7. Time response of the controlled quadrotor system flying about the hover state (ye, ze, 0)

\section{Conclusion}

The simplicity and output feedback features of the PID controllers provide significant benefits for designing efficient, low cost and flexible controllers, and the goal of this paper is to expand all these benefits to higher-order system control. A two degrees of freedom PID type controller is designed for controlling high-order dynamical systems. 
The proposed controller is constructed with optimal polynomial coefficients and solution of the Diophantine equation. The set point weights are directly incorporated into the controller design to eliminate the undesirable overshoot resulted from disturbing effects. It is shown with the numerical studies that while the controller design procedure is built upon the linearized system dynamics, the controller has a robust performance against nonlinearities, disturbances and small parameter variations. The feasibility of the proposed controller is illustrated with numerical studies on some challenging linear and nonlinear dynamical systems. The control approach can be designed for single input dynamical systems in any order to obtain superior performance. The method can be expanded to multi-input dynamical systems.

\section{REFERENCES}

[1] Y. Li, K. H. Ang, and G. Chong, "PID control system analysis and design: problems, remedies, and future directions", IEEE Control Syst. Mag., vol. 26, no. 1, pp. 32-41, 2006.

[2] K. J. Åström and T. Hägglund, "Advanced PID Control", ISA-The Instrumentation, Systems, and Automation Society, 2006.

[3] Q. Wang, Z. Ye, W. Cai, and C. Hang, PID Control for Multivariable Processes. Berlin: Springer, 2008.

[4] S. Skogestad, "Simple Analytic Rules for Model Reduction and PID Controller Tuning", J. Process Control, vol. 13, no. 1, pp. 291-309, 2003.

[5] A. S. Rao and M. Chidambaram, "Control of Unstable Processes with Two RHP Poles, a Zero and Time Delay", Asia-Pac. J. Chem. Eng., vol. 1, no. 1-2, pp. 63-69, 2006.

[6] M. Shamsuzzoha and M. Lee, "Design of Advanced PID Controller for Enhanced Disturbance Rejection of Second-Order Processes with Time Delay", AIChE J., vol. 54, no. 6 , pp. 1526-1536, 2008.

[7] J. E. Normey-Rico and E. F. Camacho, Control of Dead-time Processes, London, Springer-Verlag, 2007.

[8] M. Veronesi and A. Visioli, "Simultaneous Closed-Loop Automatic Tuning Method for Cascade Controllers", IET Control Theory Appl., vol. 5, no. 2, pp. 263-270, 2011.

[9] A. Visioli, "Design and Tuning of a Ratio Controller", Control Eng. Pract., vol. 13, no. 4, pp. 485-497, 2005.

[10] M. Araki and H. Taguchi, "Two-Degree-of-Freedom PID Controllers", Int. J. Control, vol. 1, no. 4, pp. 401-411, 2003.

[11] J. Chen and T-C Huang, "Applying Neural Networks to On-Line Updated PID Controllers for Nonlinear Process Control", J. Process Control, vol. 14, no. 2, pp. 211-230, 2004.

[12] H. A. Malki, D. Misir, D. Feigenspan, and Guanrong Chen, "Fuzzy PID Control of a Flexible-Joint Robot Arm with Uncertainties from Time-Varying Loads", IEEE Trans. Control Syst. Technol., vol. 5, no. 3, pp. 371-378, 1997.

[13] A. Visioli, Practical PID Control, London: Springer, 2006.

[14] I. Podlubny, "Fractional-Order Systems and PI-lambda D-mu Controllers", IEEE Transon Autom. Control, vol. 44, pp. 208-214, 1999.

[15] F. N. Deniz, A. Yüce, N. Tan, and D. P. Atherton, "Tuning of Fractional Order PID Controllers Based on Integral Performance Criteria Using Fourier Series Method", IFAC-Pap., vol. 50, no. 1, pp. 8561-8566, 2017.

[16] K-E Åarzén, "A Simple Event-Based PID Controller", IFAC Proc. Vol., vol. 32, no. 2, pp. 8687-8692, 1999.

[17] T. D. C. Thanh and K. K. Ahn, "Nonlinear PID Control to Improve the Control Performance of 2 Axes Pneumatic Artificial Muscle Manipulator using Neural Network", Mechatronics, vol. 16 , no. 9 , pp. $577-587,2006$.
[18] G. Ablay, "Variable Structure Controllers for Unstable Processes", J. Process Control, vol. 32, pp. 10-15, 2015.

19] A. A. Abdelrauf, M. Abdel-Geliel, and E. Zakzouk, "Adaptive PID Controller Based on Model Predictive Control", in 2016 European Control Conference (ECC), June 2016, pp. 746-751.

[20] S. Manabe and Y. C. Kim, "Recent Development of Coefficient Diagram Method", Shanghai, China, 2000.

[21] S. E. Hamamci and A. Ucar, "A Robust Model-Based Control for Uncertain Systems", Trans. Inst. Meas. Control, vol. 24, no. 5, pp. 431-445, 2002.

[22] Y-B Kang, H. Rho, and H. Kim, "A New Approach for Transient Response Control via Characteristic Ratio Assignment Method", Int. J. Control Autom. Syst., vol. 9, no. 4, p. 659, 2011.

23] M. Tabatabaei, "Generalized Characteristic Ratios: Definition, Assignment, and Application", IETE J. Res., vol. 66, no. 4, pp. $466-477,2020$.

[24] Y. C. Kim, L. H. Keel, and S. P. Bhattachayya, "Transient Response Control via Characteristic Ratio Assignment", IEEE Trans Autom. Control, vol. 48, no. 12, pp. 2238-2244, 2003.

[25] M. Haeri and M. S. Tavazoei, "CDM-Based Closed-Loop Transfer Function Design for Ramp Input", Trans. Inst. Meas. Control, vol. 33, no. 5, pp. 558-572, 2011.

[26] O. Ocal, A. Bir, and B. Tibken, "Digital Design of Coefficient Diagram Method", in Proceedings of the IEEE American Control Conference, St. Louis MO, USA, 2009, pp. 2849-2854.

[27] G. Ablay, "Coefficient Ratios-Based Robust Sliding Surface and Integral Sliding Mode Control Designs with Optimal Transient Responses", IET Control Theory Appl., vol. 8, no. 17, pp. 1896-1904, 2014.

[28] C. T. Chen, Linear System Theory and Design, New York: Oxford University Press, 1999.

[29] A. Williams and F. Taylor, Electronic Filter Design Handbook, Fourth Edition, McGraw Hill Professional, 2010.

[30] S. Manabe, "Unified Interpretation of Classical, Optimal, and Hinf Control", J. SICE, vol. 30, no. 10, pp. 941-946, 1991.

[31] M. Ben Hariz, F. Bouani, and M. Ksouri, "Robust Controller for Uncertain Parameters Systems", ISA Trans., vol. 51, no. 5, pp. 632-640, 2012.

[32] D. C. Youla, J. J. Bongiorno, and C. N. Lu, "Single-Loop Feedback-Stabilization of Linear Multivariable Dynamical Plants", Automatica, vol. 10, no. 2, pp. 159-173, 1974.

[33] A. V. Lipatov and N. I. Sokolov, "Some Sufficient Conditions for Stability and Instability of Continuous Linear Stationary Systems", Autom. Contr, vol. 39, pp. 1285-1291, 1979.

[34] N. K. Bose, E. I. Jury, and E. Zeheb, "On Robust Hurwitz and Schur Polynomials", IEEE Trans. Autom. Control, vol. 33, no. 12 , pp. $1166-1168,1988$.

[35] J. C. Lagarias, J. A. Reeds, M. H. Wright, and P. E. Wright, "Convergence Properties of the Nelder-Mead Simplex Method in Low Dimensions", SIAM J. Optim., vol. 9, pp. 112-147, 1998.

[36] F. G. Martins, "Tuning PID Controllers using the ITAE Criterion", Int J. Eng. Educ., vol. 21, no. 5, pp. 867-873, 2005.

[37] A. Tonoli, A. Bonfitto, M. Chiaberge, M. Silvagni, L. D. Suarez, and E. Zenerino, "Feedforward and Modal Control for a Multi Degree of Freedom High Precision Machine", in Smart Actuation and Sensing Systems - Recent Advances and Future Challenges, Croatia: InTech, 2012, pp. 513-546.

[38] P. Cortés-Antonio, F. Valdez, O. Castillo, and P. Melin, "Towards Tracking Trajectory of Planar Quadrotor Models", in Intuitionistic and Type-2 Fuzzy Logic Enhancements in Neural and Optimization Algorithms: Theory and Applications, Springer International Publishing, 2020, pp. 313-323.

Received 29 March 2021 